\title{
Melanoma educates mesenchymal stromal cells towards vasculogenic mimicry
}

\author{
AMALIA VARTANIAN $^{1}$, SAIDA KARSHIEVA ${ }^{2}$, VLADISLAV DOMBROVSKY ${ }^{1}$ and ALEXANDER BELYAVSKY ${ }^{2}$ \\ ${ }^{1}$ Department of Experimental Diagnosis and Biotherapy of Tumors, Blokhin Russian Cancer Research Center, \\ Moscow 115478; ${ }^{2}$ Laboratory of Stem and Progenitor Cell Biology, Engelhardt Institute of Molecular Biology, \\ Russian Academy of Sciences, Moscow 119991, Russia
}

Received May 26, 2015; Accepted April 8, 2016

DOI: $10.3892 / \mathrm{ol} .2016 .4523$

\begin{abstract}
Accumulating evidence suggests that mesenchymal stromal cells (MSCs) are recruited to the tumor, and promote tumor development and growth. The present study was performed to investigate the communication between aggressive melanoma and MSCs in vasculogenic mimicry (VM). Normal human MSCs plated on Matrigel were unable to form capillary-like structures (CLSs). By contrast, MSCs co-cultured with aggressive melanoma cell lines, namely, Mel Cher, Mel Kor and Mel P, generated CLSs. Significantly, MSCs co-cultured with poorly aggressive melanoma cells, namely, Mel Me, failed to form CLSs. To identify factors responsible for $\mathrm{VM}$, the effects of vascular endothelial growth factor A (VEGFA), pro-epidermal growth factor, basic fibroblast growth factor and stromal cell-derived factor $1 \alpha$ on the formation of CLSs by MSCs were tested. VM was induced by the addition of VEGFA, whereas other cytokines were inefficient. To confirm the hypothesis that aggressive tumor cells can increase the vasculogenic ability of MSCs, a standard B16/F10 mouse melanoma test system was used. MSCs isolated from the adipose tissues of C57BL/6 mice with melanoma formed a vascular-like network on Matrigel, whereas MSCs from healthy mice failed to form such structures. This study provides the first direct evidence that melanoma tumors educate MSCs to engage in VM. The education may occur distantly. These findings offer promise
\end{abstract}

Correspondence to: Dr Amalia Vartanian, Department of Experimental Diagnosis and Biotherapy of Tumors, Blokhin Russian Cancer Research Center, Kashirskoye shosse 24, Moscow 115478, Russia

E-mail: zhivotov57@mail.ru

Abbreviations: VEGFR, vascular endothelial growth factor receptor; VM, vasculogenic mimicry; EC, endothelial cells; CLS, capillary-like structure; MSC, mesenchymal stromal cells; bFGF, basic fibroblast growth factor, SDF-1 $\alpha$, stromal cell-derived factor $1 \alpha$

Key words: vasculogenic mimicry, melanoma, mesenchymal stromal cells, C57BL/6 mice for novel therapeutic directions in the treatment of metastatic melanoma.

\section{Introduction}

In tumors, the formation of vascular channels, cords and sinuses that lack an endothelial cell (EC) lining is observed in addition to the classical angiogenesis. These structures are formed by tumor cells and possess a PAS-positive basement membrane (1). Once plated in vitro on appropriate matrices, these tumor-derived cells adopt certain EC-like properties and develop highly patterned capillary-like structures (CLSs). This vasculogenic mimicry (VM) may be considered as another mechanism by which tumor cells can obtain nutrients and oxygen to survive, particularly in less vascularized tumor areas. In accordance with this, VM has been suggested to be regulated by hypoxia (2). The occurrence of VM is relatively rare within tumors, but the presence of VM networks in these tumors correlates with the increased risk of metastasis and, therefore, a poor outcome (3).

The development and progression of tumors is the result of evolving crosstalk between a range of cell types within the tumor and the supporting tissue or tumor stroma. The expansion, invasion, metastasis and angiogenesis of the tumor is hypothesized to be modulated by mutual interactions between tumor and stromal cells through direct contact or via paracrine action. The concept of an instructive role for the bone marrow mesenchymal stromal cells (MSCs) in regulating tumor cell fate was introduced at least 30 years ago and has been validated over the past decade (4). The secretion of chemokines/ cytokines from the tumor, including stromal cell-derived factor 1 (SDF-1)/chemokine (C-X-C motif) ligand 12, hepatocyte growth factor, vascular endothelial growth factor (VEGF), tumor growth factor, basic fibroblast growth factor (bFGF), platelet-derived growth factor and interleukin-8, is known to promote MSC migration from the bone marrow to solid tumors (5). Carcinoma-associated MSCs (CA-MSCs) are non-tumorigenic, and display a normal morphological appearance and karyotype. CA-MSCs combined with tumor cells promote in vivo tumor growth more effectively than control MSCs (6). Moreover, upon prolonged exposure to tumor cell conditioned medium, MSCs activation occurs, followed by differentiation into CAFs, which become 
members of the tumor microenvironment (7). According to the study by Annabi et al, MSCs play active angiogenic roles through the regulation of novel vessel formation, stabilization and maturation (8).

Previous studies by Nico et al (9) and Scavelli et al (10) demonstrated that macrophages and mast cells contribute to the formation of neovessels in the bone marrow in active multiple myeloma through VM, and this ability proceeds in parallel to the progression of the plasma cell tumors. The involvement of bone marrow stromal cells in the mimicry process in acute leukemia has been shown by Mirshahi et al (11). We hypothesized that there may also be crosstalk between the solid tumor cells and MSCs, leading to the formation of neovessels by the MSCs.

The present study demonstrates that aggressive melanoma cells educate MSCs to adopt certain EC-like properties and develop highly patterned CLSs. This evidence provides a novel perspective into the complex interplay between stromal and vascular components in tumors.

\section{Materials and methods}

Materials. Matrigel basement membrane matrix, Growth Factor Reduced (GFR) Matrigel, VEGF, bFGF and pro-epidermal growth factor (EGF) were obtained from Becton Dickinson Labware (Bedford, MA, USA). SDF-1 $\alpha$ was purchased from R\&D Systems, Inc. (Minneapolis, MN, USA). Anti-VEGF neutralizing antibody (anti-human mouse monoclonal; cat. no. ab1316) was obtained from Abcam (Cambridge, MA, USA). Roswell Park Memorial Institute (RPMI) 1640 medium and Collagenase Type 1 were obtained from Sigma-Aldrich (St. Louis, MO, USA). Fetal bovine serum was purchased from HyClone Laboratories, Inc. (Logan, UT, USA).

Cell culture. The four melanoma cell lines (Mel Cher, Mel Kor, Mel $\mathrm{P}$ and Mel Me) were derived from the surgical specimens of patients with disseminated melanoma, who were treated at the Blokhin Russian Cancer Research Center (Moscow, Russia). The derivation and characterization of these cell lines has been described previously (12). All experiments were performed with 70-75\% confluent cells.

Human (h)MSCs from adipose tissue were kindly provided by Dr E. Solov'yova (National Research Centre Kurchatov Institute, Moscow, Russia), and cultured under hypoxic conditions $\left(5 \% \mathrm{CO}_{2}\right.$ and $\left.5 \% \mathrm{O}_{2}\right)$. The identity of hMSCs was confirmed by fluorescence-activated cell sorting analysis using positive surface markers, namely, cluster of differentiation (CD)13, CD44, CD90 and CD105, and negative markers, namely, CD34 and CD45, and by differentiation along adipogenic and osteogenic lineages. The following antibodies (all from BioLegend, San Diego, CA, USA) were used for hMSC surface phenotyping: Anti-human mouse monoclonal CD13, PE-conjugated (clone WM15; cat. no. 301703); anti-human/mouse CD44 rat monoclonal, FITC-conjugated (clone IM7; cat. no. 103005); anti-human CD90 mouse monoclonal, FITC-conjugated (clone5E10; cat. no. 328107); anti-human CD105 mouse monoclonal, FITC-conjugated (clone 43A3; cat. no. 323203); anti-human CD34 mouse monoclonal, FITC-conjugated (clone 4H11; cat. no. 316405); and anti-human CD45 mouse monoclonal, FITC-conjugated (clone HI30; cat. no. 304005). For labeling, hMSCs were resuspended at a concentration of $2 \times 10^{6}$ cells $/ \mathrm{ml}$ in $0.5 \%$ fetal bovine serum and $2 \mathrm{mM}$ EDTA in phosphate-buffered saline. The cells were incubated with dye-antibody conjugates diluted according to the manufacturer's protocols, for $40 \mathrm{~min}$ at $4^{\circ} \mathrm{C}$, and washed twice prior to the analysis. The surface phenotype of viable (propidium iodide-negative) cells was analyzed using the Gallios flow cytometer (Beckman Coulter, Indianapolis, IN, USA). MSCs from the adipose tissues of healthy mice and mice with melanoma were isolated by finely mincing the tissues with scissors and then incubating them in collagenase solution (type IA; $2 \mathrm{mg} / \mathrm{ml}$; Sigma-Aldrich) for $30 \mathrm{~min}$ at $37^{\circ} \mathrm{C}$. This was then filtered through a $40-\mu \mathrm{m}$ cell strainer (BD Biosciences, Franklin Lakes, NJ, USA), and washed twice by centrifugation at $400 \mathrm{x}$ g for $10 \mathrm{~min}$. The final cell suspension was cultured under hypoxic conditions.

Non-contact co-culture system. To set up a non-contact co-culture system, a Transwell ${ }^{\circledR}$ system (Corning Inc., Corning, NY, USA) was used. A total of $2 \times 10^{4}$ live MSCs were seeded on the surface of each tissue culture well in a 24-well plate and allowed to adhere for 4-5 h. Melanoma cells, Mel Cher, Mel Kor, Mel P or Mel Me, were seeded onto a filter (4- $\mu \mathrm{m}$ pore size), and then the filters were inserted into the wells and co-incubated for $4-5$ days at $37^{\circ} \mathrm{C}$ in a humidified atmosphere containing $5 \% \mathrm{CO}_{2}$. The two compartments shared the same culture medium through a $4-\mu \mathrm{m}$ Transwell membrane that prevented cell migration. MSCs co-cultured without melanoma cells were used as the control. Following the treatment, the ability of the MSCs to engage in CLS formation on Matrigel was tested.

CLS formation in three-dimensional culture. Matrigel $(8.7 \mathrm{mg} / \mathrm{ml})$ was thawed at $4^{\circ} \mathrm{C}$, and $100 \mu \mathrm{l}$ was added to each well of a 24-well plate and allowed to solidify for $1 \mathrm{~h}$ at room temperature. Incubation was then performed for $30 \mathrm{~min}$ at $37^{\circ} \mathrm{C}$ in a humidified $5 \% \mathrm{CO}_{2}$ incubator. Following co-culture with aggressive or poorly aggressive melanoma cells, $2 \times 10^{5}$ MSCs were seeded in complete RPMI 1640 medium onto the gel, and incubated at $37^{\circ} \mathrm{C}$ for 5-6 h. CLS formation was then analyzed. The effect of a neutralizing anti-VEGF antibody $(5 \mu \mathrm{g} / \mathrm{ml})$ on educated MSCs was also tested. The antibody was added to the cell suspension just prior to plating on Matrigel. After $18 \mathrm{~h}$ of incubation, images of the wells were captured using an inverted light microscope (Nikon Eclipse Ti-S; Nikon Corporation, Tokyo, Japan) at x20 magnification. The effect of the cytokines on CLS formation was studied on GFR Matrigel. The cytokines (all at a $10-\mu \mathrm{M}$ final concentration) were added just prior to plating the cells on GFR Matrigel, and CLS formation analysis was performed as aforementioned.

Tumor implantation. Female C57BL/6 mice ( $\mathrm{n}=12 ; 43$ days old) were housed for a week in a room maintained at $22 \pm 1^{\circ} \mathrm{C}$ with $60 \%$ relative humidity, and were provided with free access to a non-purified diet. The animal experimental protocols were approved by the Committee for Ethics of Animal Experimentation of the Blokhin Russian Cancer Research Center, and the experiments were conducted in accordance 

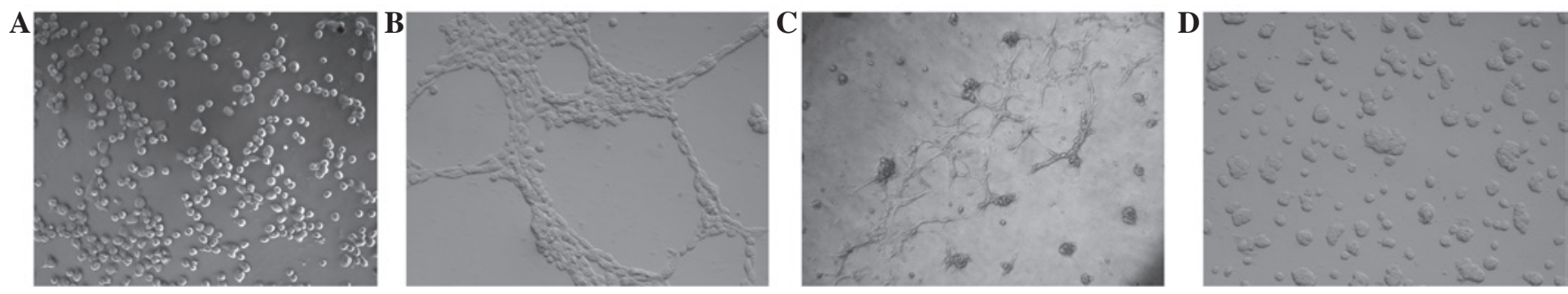

Figure 1. Melanoma cells educate hMSCs towards vasculogenic mimicry (A-D). (A) Control, non-educated hMSCs did not form CLSs on Matrigel. (B) hMSCs formed CLSs on Matrigel after co-culturing with metastatic Mel Cher melanoma cells for 5 days. (C) The CLSs formed by the hMSCs were stable for 85-90 h. (D) hMSCs did not form CLSs on Matrigel after co-culturing with non-aggressive Mel Me melanoma cells. Magnification x20. hMSCs, human mesenchymal stromal cells; CLSs, capillary-like structures.
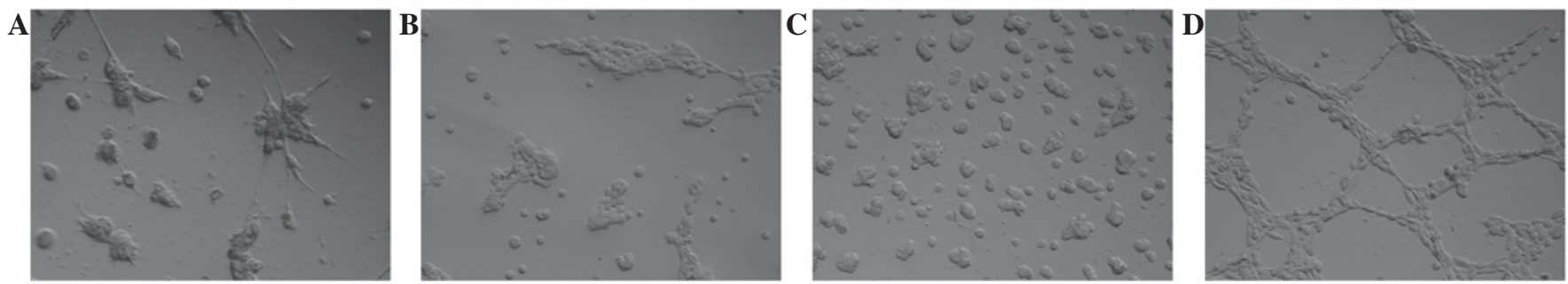

Figure 2. Role of growth factors on the formation of CLSs by hMSCs on GFR-Matrigel. Several growth factors, (A) stromal cell-derived factor 1 $\alpha$,(B) basic fibroblast growth factor, (C) pro-epidermal growth factor and VEGFA (D) were added at a concentration of $500 \mathrm{ng} / \mathrm{ml}$ to hMSCs grown on GFR-Matrigel. VEGFA significantly induced CLS formation. Magnification x20. hMSCs, human mesenchymal stromal cells; CLSs, capillary-like structures; GFR, Growth Factor Reduced; VEGFA, vascular endothelial growth factor A.

with the Guidelines for Animal Experiments in Blokhin Russian Cancer Research Center. Solid-type melanoma tumors were prepared by subcutaneous transplantation of $3 \times 10^{6} \mathrm{~B} 16 / \mathrm{F} 10$ melanoma cells $(0.3 \mathrm{ml})$ into the backs of the female mice on day 0 . On day 15 , the mice were sacrificed by cervical dislocation, and MSCs from the adipose tissues of healthy mice and mice with melanomas were isolated. The ability of these cells to form CLSs on Matrigel was tested, as previously described.

\section{Results}

Highly aggressive melanoma cells educate MSCs to form CLSs. Based on the analogy with the results of Mirshahi et al demonstrating the generation of VM channels by MSCs in acute leukemic bone marrow (11), we hypothesized that there may be a similar crosstalk between the aggressive melanoma cells and MSCs resulting in acquisition by MSCs of features required for tumor VM. To confirm this, a Transwell co-culture system was utilized in the present study. Normal human MSCs plated on Matrigel were unable to form CLSs (Fig. 1A). However, MSCs co-cultured with highly aggressive Mel Cher melanoma cells for 5 days spontaneously generated CLSs (Fig. 1B). The CLSs formed by the MSCs remained stable for $>3.5$ days. The penetration of CLSs into the matrix was observed (Fig. 1C). Significantly, MSCs failed to form CLSs if grown with poorly aggressive Mel Me melanoma cells (Fig. 1D).

Effect of angiogenic growth factors on CLS formation by educated MSCs. To identify the factors that are responsible for CLS formation by human MSCs, the effects of the addition of
SDF-1 $\alpha$, VEGFA, bFGF and EGF were tested. The contribution of all four cytokines in angiogenesis has been repeatedly reported during the last decade. On the basis of the observations showing SDF-1 $\alpha$ to be one of the most prominent candidates involved in the formation of CLSs (13), the involvement of this cytokine was expected in CLS formation in the present study. However, in the present experiments, occasional clusters of cells were observed, and some cells were abnormally elongated in response to SDF-1 $\alpha$, but CLSs were not found in GFR Matrigel (Fig. 2A). bFGF and EGF were also inactive in inducing the formation of CLSs by MSCs in this system (Fig. 2B and C). In contrast to the aforementioned factors, robust CLS formation in GFR Matrigel was induced by VEGFA (Fig. 2D).

Education of MSCs by metastatic melanoma B16/F10 in vivo. Pilot in vitro experiments showed that B16/F10 mouse melanoma cells can educate mouse MSCs towards a VM. To further test the hypothesis that aggressive melanoma educates MSCs towards a VM, in vivo experiments using a standard B16/F10 mouse melanoma test system were performed. MSCs isolated from the adipose tissues of healthy C57BL/6 mice were unable to form CLSs on Matrigel (Fig. 3A). By contrast, MSCs isolated from the adipose tissues of mice with melanoma formed CLSs under similar conditions (Fig. 3B). It is worth noting that the educated MSCs retained their ability to form CLSs after an additional two passages.

Next, the study attempted to verify whether VEGFA was the primary factor triggering CLS formation by educated MSCs. When MSCs isolated from the adipose tissues of mice with melanoma were treated with VEGF neutralizing antibody and then seeded on Matrigel, no early signs of CLS formation were observed (Fig. 3C). 

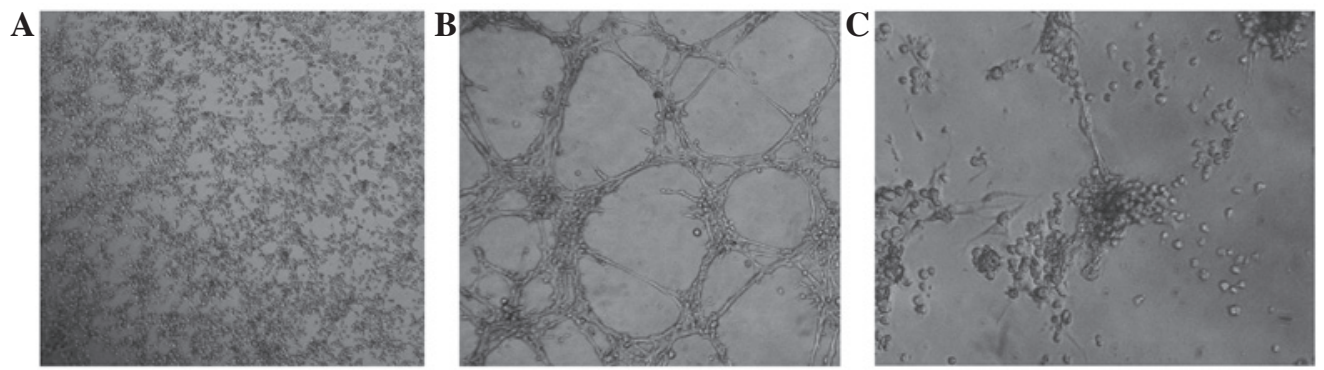

Figure 3. Melanoma B16/F10 tumors educate MSCs to form CLSs on Matrigel. (A) MSCs from the adipose tissues of healthy C57BL/6 mice did not form CLSs on Matrigel. (B) MSCs from the adipose tissues of mice with melanoma formed CLSs on Matrigel. (C) Inhibition of CLS formation by an anti-vascular endothelial growth factor A neutralizing antibody. Magnification x20. MSCs, mesenchymal stromal cells; CLSs, capillary-like structures.

\section{Discussion}

In our previous study, it was shown that disseminated melanoma adopted a vascular-like phenotype and engaged in VM (14), as similarly found in previous observations in uveal melanoma. In other previous studies, we also identified certain molecular determinants that drive the expression of a latent 'vasculogenic program' that signals CLS formation in melanoma cells, which is a hallmark of tumor VM $(15,16)$. The present study demonstrated that i) highly aggressive melanoma cells have the potential to educate MSCs to adopt certain EC-like properties and develop highly patterned CLSs; ii) robust CLS formation is induced by VEGFA; and iii) MSCs isolated from the adipose tissues of mice with melanoma form CLSs.

EC proliferation causes new blood vessels to arise from the preexisting vasculature. However, the recruitment of bone marrow-derived endothelial progenitor cells also mediates tumor vascularization. Upon histomorphological analysis, certain tumors were shown to be vascularized by pre-existing vessels, without significant angiogenesis; this process is termed 'vascular co-option'. Tumors can also be vascularized via the formation of vascular channels with the tumor's own cells in a non-EC process termed 'vasculogenic mimicry'. It has been shown that mast cells or macrophages participate in vessel wall formation in biopsy specimens of patients with active multiple myeloma. The two cell populations retain their lineage markers and can be regarded as cells that do not transdifferentiate into ECs. Similar to macrophages and mast cells in active multiple myeloma, in an ovarian tumor, Conejo-Garsia et al found a population of CD45-positive/vascular endothelial cadherin-positive cells, the so-called 'vascular leukocytes', which displayed the morphological and functional properties of endothelial-like cells, with the ability to contribute functionally to vasculogenesis in vivo (17). In the present study, it was shown that highly aggressive melanoma Mel Cher cells may have the capacity to educate MSCs to form CLSs through the regulation of their angiogenic properties (Fig. 1A and B). Our previous study showed that CLSs formed by melanoma cells on Matrigel were stable 20-24 h after seeding the cells onto matrix; thereafter the cell junctions characteristic of the CLSs were lost and the spontaneous release of focal contacts occurred (14). In the present study, the CLSs formed by MSCs remained stable for $>3.5$ days. Moreover, the penetration of CLSs into the matrix could be observed (Fig. 1C). The results found with two other aggressive melanoma cell lines, Mel
Kor and Mel P, extended the observation that melanoma cells educate MSCs towards VM (data not shown). In addition, the MSCs failed to form CLSs when grown with poorly aggressive melanoma Mel Me cells (Fig. 1D), suggesting that poorly aggressive melanoma cells lack the capacity to account for CLS formation by MSCs.

It is now established that tumor cells secrete chemoattractant factors that facilitate MSC homing to the tumors (18). However, MSCs secrete factors that stimulate tumor cell proliferation, suggesting the existence of contact-independent interactions between MSCs and cancer cells in vivo (19). In addition, in a non-contact co-culture system, EC proliferation and migration is increased by MSCs, thus promoting early angiogenic events. One of the most prominent candidates to be involved in the formation of CLSs by MSCs is SDF-1 $1 \alpha$. This chemokine induces an enhanced connection between embryonic stem cells, thereby increasing the ability of the cells to assemble in a reticular network (13). SDF-1 $\alpha$ also enhances vasculogenesis by recruiting vascular progenitors to the neovasculature (20). However, in the present experiments, VEGFA was sufficient to induce CLSs on the GFR Matrigel by educated MSCs, whereas bFGF, EGF and SDF-1 $\alpha$ were inactive. VEGFA involvement in the mimicry process was confirmed by adding a neutralizing antibody against VEGF. Anti-VEGF antibody abrogated CLS formation. This adds to the evidence that VEGF may control MSC vasculogenesis. Our previous study (15) and studies by other groups (21) have shown that tumor VM is under the control of the VEGF/VEGFR1 signaling pathway, while the VEGF/VEGFR2 signaling pathway plays a crucial role in the survival, proliferation and differentiation of ECs and their progenitors. MSCs have been shown to express high level of VEGFR1 and a lower level of VEGFR2 on the cell surface. Moreover, hypoxia has been shown to increase the expression of VEGFR1 (22). These data may thus explain the dependence of CLS formation by MSCs on VEGFA.

To confirm the hypothesis of a novel qualitative signature of MSCs to support tumor vascularization through crosstalk with aggressive tumors, in vivo animal testing was also performed in the present study. A pilot experiment using a standard B16/F10 mouse melanoma test system showing the same CLS-forming behavior as the human cells lines in the study was performed. The data show for the first time that aggressive melanoma educates MSCs towards VM.

Tumor vascularization is known to be critical for tumor growth, since sufficient nutrients and oxygen must be supplied to cancer cells to ensure their survival and proliferation. VM 
has already been described in a number of tumors, and it has also been shown that VM contributes to the delivery of blood to tumors due to connection of vascular channels with regular blood vessels. The present data indicated that MSCs may acquire the capacity to participate in melanoma vascularization through regulation of their angiogenic properties. The present study did not investigate whether vascular channels formed by MSCs could contribute to the blood supply of tumors in vivo. However, the evidence presented highlights the importance of the stromal microenvironment during vascularization of melanoma and may provide clues for the development of effective strategies for MSC-based cancer therapies.

\section{Acknowledgements}

This study was supported by a grant (no. 14-35-00107) from the Russian Science Foundation. The authors would like to thank Dr E. Solov'yova (National Research Centre Kurchatov Institute) who kindly provided the hMSCs used in this study, and Dr M. Krasil'nikov (Department of Cancerogenesis, Blokhin Russian Cancer Research Center) for valuable comments and advice. The authors are also grateful to Mr. A. Sherbakov (Department of Experimental Diagnosis and Biotherapy of Tumors, Blokhin Russian Cancer Research Center) for administering technical assistance.

\section{References}

1. Maniotis AJ, Folberg R, Hess A, Seftor EA, Gardner LM Pe'er J, Trent JM, Meltzer PS and Hendrix MJ: Vascular channel formation by human melanoma cells in vivo and in vitro: Vasculogenic mimicry. Am J Pathol 155: 739-752, 1999.

2. Sun B, Zhang D, Zhang S, Zhang W, Guo H and Zhao X: Hypoxia induces vasculogenic mimicry channel formation and tumor invasion-related proteins expression in melanoma. Cancer Lett 249: 188-197, 2007.

3. Paulis YW, Soetekouw PM, Verheul HM, Tjan-Heijnen VC and Griffioen AW: Signaling pathways in vasculogenic mimicry. Biochem Biophys Acta 1806: 18-28, 2010.

4. Mishra PJ, Mishra PJ, Glod JW and Banerjee D: Mesenchymal stem cells: Flip side of the coin. Cancer Res 69: 1255-1258, 2009.

5. Studeny M, Marini FC, Dembinski JL, Zompetta C, Cabreira-Hansen M, Bekele BN, Champlin RE and Andreeff M: Mesenchymal stem cells: Potential precursors for tumor stroma and targeted-delivery vehicles for anticancer agents. J Natl Cancer Inst 96: 1593-1603, 2004.

6. McLean K, Gong Y, Choi Y, Deng N, Yang K, Bai S, Cabrera L, Keller E, McCauley L, Cho KR and Buckanovich RJ: Human ovarian carcinoma-associated mesenchymal stem cells regulate cancer stem cells and tumorigenesis via altered BMP production. J Clin Invest 121: 3206-3219, 2011.
7. Barcellos-de-Souza P, Gori V, Bambi F and Chiarugi P: Tumor microenvironment: Bone marrow-mesenchymal stem cells as key players. Biochim Biophys Acta 1836: 321-335, 2013.

8. Annabi B, Lee YT, Turcotte S, Naud E, Desrosiers RR, Champagne M, Eliopoulos N, Galipeau J and Béliveau R: Hypoxia promotes murine bone-marrow-derived stromal cell migration and tube formation. Stem Cells 21: 337-347, 2003.

9. Nico B, Mangieri D, Crivellato E, Vacca A and Ribatti D: Mast cells contribute to vasculogenic mimicry in multiple myeloma. Stem Cell Dev 17: 19-22, 2008.

10. Scavelli C, Nico B, Cirulli T, Ria R, Di Pietro G, Mangieri D, Bacigalupo A, Mangialardi G, Coluccia AM, Caravita T, et al: Vasculogenic mimicry by bone marrow macrophages in patients with multiple myeloma. Oncogene 27: 663-674, 2008.

11. Mirshahi P, Rafii A, Vincent L, Berthaut A, Varin R, Kalantar G, Marzac C, Calandini OA, Marie JP, Soria C, et al: Vasculogenic mimicry of acute leukemic bone marrow stromal cells. Leukemia 23: 1039-1048, 2009.

12. Mikhailova IN, Lukashina MI, Baryshnikov AY, Morozova LF, Burova OS, Palkina TN, Kozlov AM, Golubeva VA, Cheremushkin EA, Doroshenko MB, et al: Melanoma cell lines as the basis for antitumor vaccine preparation. Vestn Ross Akad Med Nauk: 37-40, 2005 (In Russian).

13. Chen T, Bai H, Shao Y, Arzigian M, Janzen V, Attar E, Xie Y, Scadden DT and Wang ZZ: Stromal cell-derived factor-1/CXCR4 signaling modifies the capillary-like organization of human embryonic stem cell-derived endothelium in vitro. Stem Cells 25: 392-401, 2007.

14. Vartanian A, Burova O, Stepanova E, Baryshnikov A and Lichinitser MR: The involvement of apoptosis in melanoma vasculogenic mimicry. Melanoma Res 17: 1-8, 2007.

15. Vartanian A, Stepanova E, Grigorieva I, Solomko E, Baryshnikov A and Lichinitser M: VEGFR1 and PKC $\alpha$ signaling control melanoma vasculogenic mimicry in a VEGFR2 kinase-independent manner. Melanoma Res 21: 91-98, 2011.

16. Vartanian A, Gatsina G, Grigorieva I, Solomko E, Dombrovsky V, Baryshnikov A and Stepanova E: The involvement of Notch signaling in melanoma vasculogenic mimicry. Clin Exp Med 13: 201-219, 2013.

17. Conejo-Garcia JR, Buckanovich RJ, Benencia F, Courreges MC, Rubin SC, Carroll RG and Coucos G: Vascular leukocytes contribute to tumor vascularization. Blood 105: 679-681, 2005.

18. Reagan MR and Kaplan DL: Concise review: Mesenchymal stem cell tumor-homing: Detection methods in disease model systems. Stem Cells 29: 920-927, 2011.

19. Hou L, Wang X, Zhou Y, Ma H, Wang Z, He J, Hu H, Guan W and Ma Y: Inhibitory effect and mechanism of mesenchymal stem cells on liver cancer cells. Tumour Biol 35: 1239-1250, 2014.

20. Aghi M, Cohen KS, Klein RJ, Scadden DT and Chiocca EA: Tumor stromal-derived factor-1 recruits vascular progenitors to mitotic neovasculature, where microenvironment influences their differentiated phenotypes. Cancer Res. 66: 9054-9064, 2006.

21. Wang JY, Sun T, Zhao XL, Zhang SW, Zhang DF, Gu Q, Wang XH, Zhao N, Qie S and Sun BC: Functional significance of VEGF-a in human ovarian carcinoma: Role in vasculogenic mimicry. Cancer Biol Ther 7: 758-766, 2008.

22. Okuyama H, Krishnamachary B, Zhou YF, Nagasawa H, Bosch-Marce M and Semenza GL: Expression of vascular endothelial growth factor receptor 1 in bone marrow-derived mesenchymal cells is dependent on hypoxia-inducible factor 1 . J Biol Chem 281: 15554-15563, 2006. 